\title{
Development of Blood and Lymphatic Endothelial Cells in Embryonic and Fetal Human Skin
}

Christopher Schuster, ${ }^{*}$ Michael Mildner, ${ }^{\dagger}$ Albert Botta, ${ }^{*}$ Lucas Nemec, ${ }^{*}$ Radu Rogojanu ${ }^{\ddagger \S}{ }^{\ddagger}$ Lucian Beer ${ }^{\dagger}$ Christian Fiala, ${ }^{\oplus}$ Wolfgang Eppel,, Wolfgang Bauer, ${ }^{*}$ Peter Petzelbauer, ${ }^{* *}$ and Adelheid Elbe-Bürger*

From the Laboratory of Cellular and Molecular Immunobiology of the Skin, * Division of Immunology, Allergy and Infectious Diseases, the Research Divisions of Biology and Pathobiology of the Skin ${ }^{\dagger}$ and Skin \& Endothelium, ** Department of Dermatology, and the Departments of Pathophysiology and Allergy Research ${ }^{\S}$ and Gynaecology and Obstetrics, ${ }^{\|}$Medical University of Vienna, Vienna; the TissueGnostics GmbH, ${ }^{\ddagger}$ Vienna; and the Gynmed-Ambulatorium, ${ }^{\circledR}$ Vienna, Austria

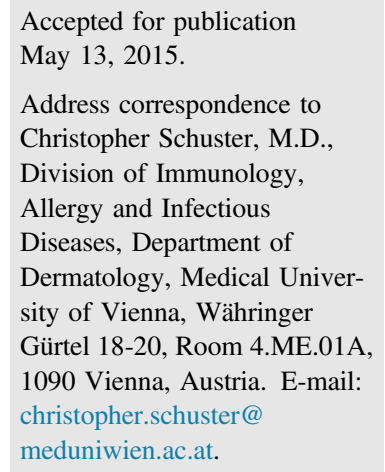

\begin{abstract}
Blood and lymphatic vessels provide nutrients for the skin and fulfill important homeostatic functions, such as the regulation of immunologic processes. In this study, we investigated the development of blood and lymphatic endothelial cells in prenatal human skin in situ using multicolor immunofluorescence and analyzed angiogenic molecules by protein arrays of lysates and cell culture supernatants. We found that at 8 to 10 weeks of estimated gestational age, $\mathrm{CD}_{144^{+}}$vessels predominantly express the venous endothelial cell marker PAL-E, whereas CD144 ${ }^{+}$PAL- $E^{-}$vessels compatible with arteries only appear at the end of the first trimester. Lymphatic progenitor cells at 8 weeks of estimated gestational age express CD31, CD144, Prox1, and temporary PAL-E. At that developmental stage not all lymphatic progenitor cells express podoplanin or Lyve-1, which are acquired with advancing gestational age in a stepwise fashion. Already in second-trimester human skin, the phenotype of blood and lymphatic vessels roughly resembles the one in adult skin. The expression pattern of angiogenic molecules in lysates and cell culture supernatants of prenatal skin did not reveal the expected bent to proangiogenic molecules, indicating a complex regulation of angiogenesis during ontogeny. In summary, this study provides enticing new insights into the development and phenotypic characteristics of the vascular system in human prenatal skin. (Am J Pathol 2015, 185: 2563-2574; http://dx.doi.org/10.1016/j.ajpath.2015.05.006)
\end{abstract}

Human skin is a highly vascularized tissue that contains both blood vessels (BVs) and lymphatic vessels (LVs) that initially form during intrauterine life to give rise to anatomically and phenotypically distinct, nonanastomizing networks. ${ }^{1-7}$ In healthy adult skin, the blood vascular network provides oxygen and nutrients for the skin and plays a central role in controlling homeostatic immunologic functions, including the immigration of leukocytes. The lymphatic system, by contrast, drains excessive interstitial fluid and serves as a conduit for transporting antigen-presenting cells and $\mathrm{T}$ cells to regional lymph nodes. The endothelial cells (ECs) lining both BVs and LVs express the pan-EC markers CD144 (VE-cadherin), CD31, and CD34. ${ }^{1,8}$ PAL-E is currently the most specific marker for venous and blood endothelial cells (BECs), ${ }^{9,10}$ whereas Lyve-1, Prox1, and podoplanin are used to characterize lymphatic endothelial cells (LECs). ${ }^{6,8}$ Owing to their diverse functions, BVs and LVs also vary in the structure of their vascular basement membrane and the content of accessory molecules. BVs in general contain more collagen type IV (CIV) than LVs in their basement membrane, and smooth muscle actin (SMA) ${ }^{+}$cells are found in the wall of various BVs, such as arterioles and venules, but not in smaller LVs. ${ }^{7}$ Although the functional consequence of different levels of accessory molecules remains to be elucidated, the major histocompatibility complex class II molecule HLA-DR is expressed on BECs but not on LECs, whereas the scavenger and thrombospondin receptor CD36 is found on LECs and a subset of BECs. ${ }^{1,11-14}$

During intrauterine life, cutaneous ECs form the complex array of vessels differing markedly in structure and function to eventually give rise to the blood and lymphatic system reminiscent of adult skin. EC-forming tube-like structures

Supported by Austrian Science Fund grants P19474-B13 (A.E.-B) and DK W1248-B13 (Winfried Pickl and A.E.-B) and Austrian National Bank grant 15427 (P.P.).

Disclosures: None declared. 
have been described in the human embryonic dermis as early as 6 weeks of estimated gestational age (EGA). ${ }^{15}$ They are surrounded by an immature, rudimentary basement membrane already containing some integral components, such as CIV and laminin, ${ }^{16,17}$ and are initially organized in one and, after 8 weeks of EGA, in two planes parallel to the primitive epidermis. ${ }^{15}$ ECs were identified at these early developmental stages based on routine histologic analysis, electron microscopy, and expression of the blood EC markers factor VIII-related antigen and CD34. ${ }^{15,18-20}$ The expression profile of accessory molecules on prenatal and adult EC differs, exemplified by either the reduced levels of vascular adhesion molecules (CD62P, CD54) and HLA$\mathrm{DR}^{11,19}$ or the presence of molecules not found in healthy adult skin, such as CD105 or CD206, on ECs in developing skin. $^{21,22}$ The differentiation of LECs using markers such as Prox1, Lyve-1, and podoplanin was first described at approximately 11 weeks of EGA, the earliest developmental time point investigated. ${ }^{23,24}$

Previous studies investigating the development of human cutaneous vasculature largely do not distinguish between BECs and LECs and are inhomogeneous with regard to the markers studied and the developmental age investigated, often starting their analysis at the end of the first trimester. ${ }^{11,15-24}$ Thus, to better understand the formation of the cutaneous vascular system, we investigated the quantitative and phenotypic development of BVs and LVs using a panel of well-established antibodies identifying all (CD31, CD34, CD144) or BECs (PAL-E) and LECs (Lyve-1, Prox1) only and analyzed angiogenic factors that might regulate their maturation during intrauterine life.

\section{Materials and Methods}

\section{Skin Samples}

After legal termination of pregnancy, 28 specimens of human embryonic and fetal trunk skin ranging from 8 to 24 weeks of EGA were studied. The age was estimated by crown-rump length and maternal history. Healthy adult (19 to 59 years, 10 donors) skin was collected after abdominal, back, and breast surgery. The study was approved by the local ethics committee and conducted in accordance with the principles of the Declaration of Helsinki. Parents and participants gave their written informed consent.

\section{Immunofluorescence}

Skin specimens were embedded in optimum cutting tissue compound (Tissue-Tek; Sakura Finetek, Zoeterwoude, the Netherlands), snap frozen in liquid nitrogen, and stored at $-80^{\circ} \mathrm{C}$ until further processing. Seven-micrometer sections were cut, air dried, fixed in ice-cold acetone (CD34, $\mathrm{CIV}$, or laminin) or $4 \%$ paraformaldehyde at ambient temperature for 10 minutes, and washed in phosphate-buffered saline. Fixed sections were stained with unconjugated antibodies [CD68 (KP1; Dako, Glostrup, Denmark), CD144 (TEA 1/31; Beckmann Coulter, Fullerton, CA), CD163 (ED2; Acris, Herford, Germany), CIV (rabbit polyclonal; Abcam, Cambridge, UK), laminin (4C7; Dako), Lyve-1 (rabbit polyclonal; Acris), PAL-E (PAL-E; Fitzgerald Industries, North Acton, MA), podoplanin (18H5; eBioscience, San Diego, CA), Prox1 (polyclonal goat IgG; R\&D Systems, Minneapolis, MN), or SMA (1A4, Dako)] or conjugated primary antibodies [biotin-conjugated CD144/VEcadherin (polyclonal goat IgG; R\&D Systems) or fluorescein isothiocyanate (FITC)-anti-CD34 (AC136; Miltenyi)] overnight at $4^{\circ} \mathrm{C}$ and then detected with donkey-anti-mouse NorthernLight 637 (R\&D Systems), Alexa Fluor 488 goatanti-mouse, Alexa Fluor 488 goat-anti-rabbit, Alexa Fluor 546 goat-anti-rabbit, Alexa Fluor 546 goat-anti-mouse, or Alexa Fluor 555 streptavidin (all Life Technologies, Carlsbad, CA). Subsequently, sections that included a second mouse antibody were blocked with $10 \%$ mouse serum and $2 \%$ mouse IgG (BD Bioscience, San Jose, CA) for 1 hour at room temperature and stained with the following conjugated antibodies overnight at $4^{\circ} \mathrm{C}$ : FITC-anti-HLA-DR (L243, BD Biosciences), FITC-anti-CD31 (WM59, Serotec, Kidlington, UK), FITC-anti-CD36 (TR9, Immunotools, Friesoythe, Germany), and Alexa Fluor 555-anti-CD45 (MEM28; Exbio, Vestec, Czech Republic). Sections stained with Prox1 antibodies were detected using biotin-conjugated rabbit anti-goat IgG [Elite Goat Vectastain Kit (Vector Laboratories, Burlingame, CA)] and subsequently visualized using Alexa Fluor 555 streptavidin (Life Technologies) or NorthernLight 637 streptavidin (R\&D Systems). For CD68 and CD163 staining, a nuclear counterstain was performed using DAPI (Roche, Mannheim, Germany). Slides were mounted using Vectashield (Vector Laboratories), and images were recorded using a confocal laser scanning microscope (LSM 410; Carl Zeiss, Inc., Jena, Germany) equipped with four lasers emitting lights at $405,488,543$, and $633 \mathrm{~nm}$.

\section{Preparation of Skin Single-Cell Suspensions and Lysates for Protein Array Analysis}

Single-cell suspensions from prenatal (11 to 24 weeks of EGA) and adult skin (19 to 46 years of age) were prepared as previously described. ${ }^{11}$ After 48 hours of culture, supernatants were snap frozen and stored at $-80^{\circ} \mathrm{C}$ until use. Lysates from prenatal (11 to 24 weeks of EGA) and adult skin (44 to 51 years of age) were prepared from optimum cutting tissue compoundembedded skin samples that were thawed and washed three times in phosphate-buffered saline to remove the optimum cutting tissue compound. The samples were homogenized by sonication in $1 \%$ Triton $\mathrm{X}$ lysis buffer that contained complete protease inhibitors (Roche). After centrifugation, the protein concentration was measured using the BCA Kit (Life Technologies). Aliquots containing $200 \mu \mathrm{g}$ of total protein and cell culture supernatants were analyzed by the Angiogenesis Proteome Profiler Kit (Proteome Profiler Arrays; R\&D Systems) according to the manufacturer's instructions. 
Quantification of Vessel Density and Size, Analysis of Marker Co-Expression in Skin Sections, and Statistical Analysis

For the quantification of vessel density and size, 60 to 340 highpower fields with expression of CD144 were imported into the data analysis software StrataQuest version 5.0 (TissueGnostics, Vienna, Austria), an improved version of TissueQuest version 4.0. ${ }^{25}$ One analysis layer calculated the tissue area after the subtraction of the epidermis, whereas the second analysis layer determined each individual CD144 ${ }^{+}$vessel, including its respective size. Manual correction tools enabled quick review and adjustments of the vessel-labeled masks using brushes of configurable sizes and in-painting procedures. Thus, by fusing broken vessel walls, a correct total count was ensured. Extracted Microsoft Excel files with sample statistics and raw data were further processed in GraphPrism version 5.03 (GraphPad Software, San Diego, CA). Differences between groups were assessed with the $t$-test (GraphPad Software). The reported $P$ value is a result of a two-sided test. $P<0.05$ was considered statistically significant.

The co-expression of Prox 1, Lyve-1, and podoplanin reactive LVs was quantified on triple-stained sections by two independent investigators (C.S. and L.N.) in 28 to 88 high-power fields. The staining intensity of the respective LV markers was not considered in the subsequent statistical analysis. For the investigation of the anatomical distribution of LVs, the dermis was divided into upper, middle, and lower thirds; the number of LVs in each compartment was counted in Prox1, Lyve-1, and podoplanin triple-stained sections by two independent investigators (C.S. and L.N.); and finally, the relative LV number in each compartment was calculated. This approach (ie, comparing the relative position of LVs in the dermis) is necessary given the marked differences in the anatomy of prenatal skin compared with adult skin, such as the absence of the papillary dermis until midgestation and the different dermal thickness. Differences between groups were assessed with the $t$ test (GraphPad Software).

Co-expression of PAL-E and Lyve-1 on CD144 ${ }^{+}$vessels and expression of HLA-DR or CD36 on BVs and LVs was analyzed by two independent investigators (C.S. and A.B.) in 23 to 96 high-power fields (Carl Zeiss). Expression of PAL-E, Lyve-1, HLA-DR, or CD36 was considered positive when their staining largely covered the CD144 staining. The intensity of the staining was not considered for the subsequent statistical analysis. Differences between groups were assessed with the $U$ test (GraphPad Software).

\section{Results}

CD144 Is Superior to CD34 as a pan-EC Marker in Both Prenatal and Adult Human Skin

To uncover the ideal pan-EC marker for subsequent experiments, we performed immunofluorescence staining in prenatal and, for comparison, healthy adult human skin. We found that in adult skin all CD34 ${ }^{+}$vessels express CD144 (Figure 1A and Supplemental Figure S1) and that some CD144 ${ }^{+}$vessels with lymphatic morphologic features do not or weakly express CD34 (Figure 1A). ${ }^{1,8,12}$ Additional nuclear staining with Prox1 unanimously identifies these vessels as LVs (Figure 1A). This staining pattern (ie, $\mathrm{CD} 144^{+}$outnumbering $\mathrm{CD} 34^{+}$vessels and $\mathrm{CD} 34^{\text {weak/ }}$ - vessels expressing Prox1) is identical in prenatal human skin, with the exception that no vessels are detected in a small subepidermal band and that the frequency of $\mathrm{CD} 144^{+} \mathrm{CD} 34^{-}$vessels is diminished (Figure 1A and Supplemental Figure S1). Although CD144 is exclusively expressed on vessels, CD34 can be identified, apart from the vasculature, in all layers of prenatal skin. Consequently, we opted for CD144 as the ideal marker for subsequent experiments given the exclusive vascular staining pattern and the resulting superior signal-to-noise ratio.

The frequency of $\mathrm{CD} 144^{+}$vessels in prenatal skin in all developmental stages investigated is significantly higher than in adult skin, probably accounting for the higher nutrient requirements of the growing skin (Figure 1B) ${ }^{26}$ Although the mean vessel size is significantly lower between 8 and 10 weeks of EGA than in adult skin, no significant difference can be detected after 11 weeks of EGA as the mean size increases (Figure 1C).

\section{CD144 ${ }^{+}$PAL-E ${ }^{+}$Vessels Are Predominant in Embryonic Skin}

The molecular heterogeneity of ECs forms the basis of the different functions of BVs and LVs. To gain insight into potentially associated functions and their origin, we performed kinetics studies of BVs and LVs using triple immunofluorescence staining on embryonic, fetal, and adult skin sections. In addition to CD144, we used PAL-E as a marker for EC lining capillaries and small veins but not arteries or $\mathrm{LVs}^{9,10}$ as well as the $\mathrm{LV}$ antigen Lyve-1, which is also expressed by some tissue macrophages. ${ }^{8,27-29}$ We identified three distinct populations in adult skin: $\mathrm{CD} 144^{+} \mathrm{PAL}-\mathrm{E}^{+}$ capillaries and veins, $\mathrm{CD} 144^{+} \mathrm{PAL}^{-} \mathrm{E}^{-}$arteries, and CD144 ${ }^{+}$Lyve- ${ }^{+}$LVs (Figure 1D and Supplemental Figure S2). In contrast, the composition of vessels in embryonic skin (8 to 10 weeks of EGA) differs significantly from adult skin. Most CD144 ${ }^{+}$vessels colocalize with PAL$\mathrm{E}$, whereas almost no $\mathrm{CD} 144^{+} \mathrm{PAL}_{-} \mathrm{E}^{-}$arterial ECs are detectable, pointing to a very early and immature EC phenotype and perhaps the, at least phenotypic, impending separation of veins and arteries (Figure 1, D and E). In five of nine samples, few CD144 ${ }^{+}$Lyve- ${ }^{+}$LVs were detected; intriguingly, some of these vessels also weakly co-expressed PAL-E (Figure 1D and Supplemental Figure S2), representing either an activated BEC phenotype or a possible BECLEC-transdifferentiation. ${ }^{30}$

Next, we investigated whether PAL- ${ }^{\text {weak }}$ vessels in firsttrimester human skin express Prox 1, the master regulator of LV development and the first known step in the acquisition of LEC identity. ${ }^{31} \mathrm{We}$ found that a small fraction of CD31 ${ }^{+}$ 

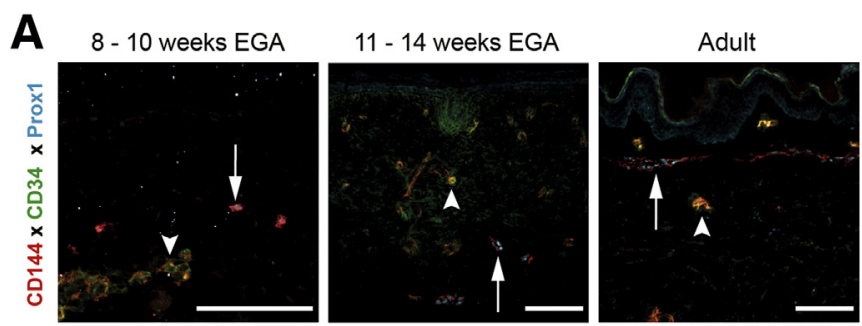

B



C

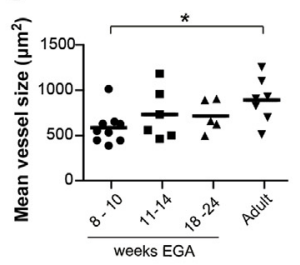

D
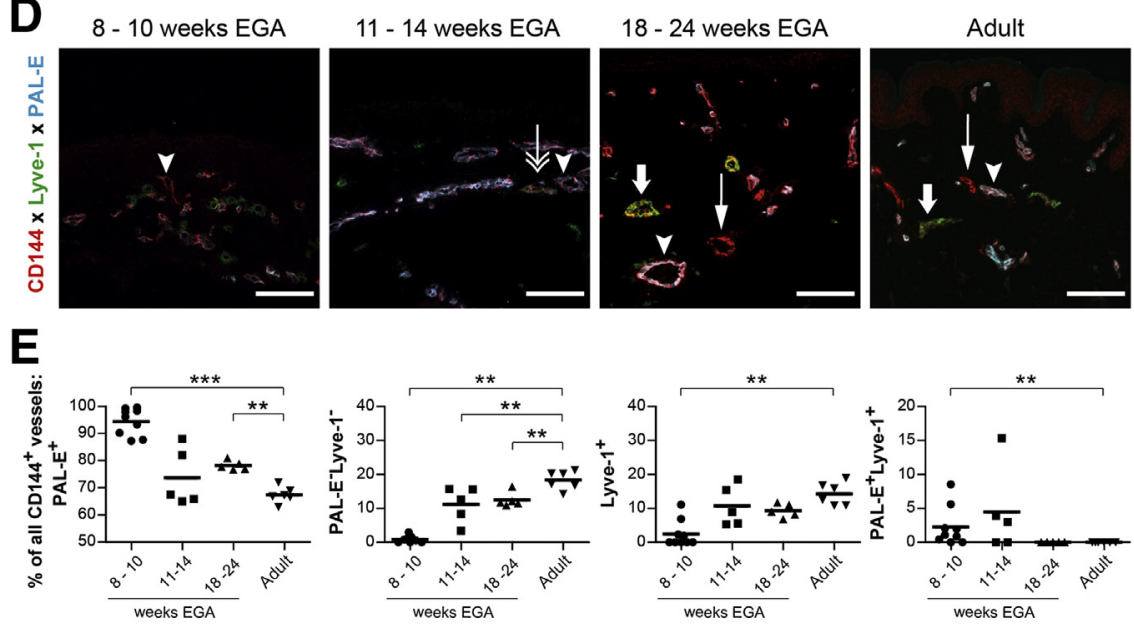

$\mathbf{F}$
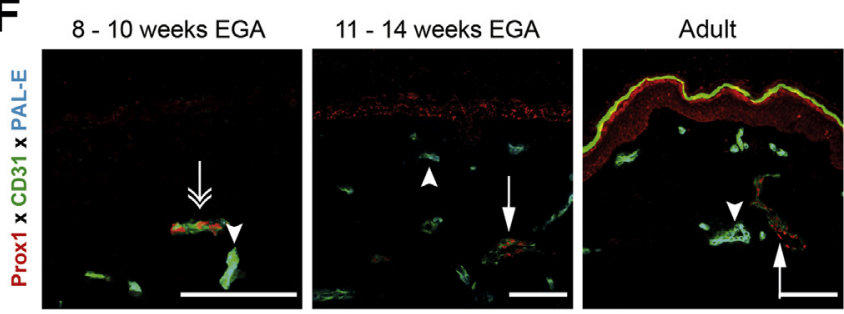

vessels between 8 to 10 weeks of EGA but not at later stages of development express both PAL-E and Prox 1 (Figure 1F and Supplemental Figure S3). This mixed phenotype (ie, the combination of the master regulator of lymphatic development and the most important venous EC marker) perhaps favors the notion of LEC transdifferentiation from BEC at that early developmental stage.

In addition, CD144- ${ }^{-}$yve- $1^{+}$single cells can be identified at higher frequencies than in adult skin. These cells always co-express typical macrophage markers, such as the haptoglobin receptor CD163 (Supplemental Figure S4) and CD68 (data not shown). ${ }^{32}$ Thus, CD144- ${ }^{-} y v e 1^{+} \mathrm{CD} 163^{+}$ or $\mathrm{CD} 144^{-} \mathrm{Lyve}^{+} \mathrm{CD}^{+} 8^{+}$single cells represent a macrophage subset, given that not all $\mathrm{CD} 163^{+}$or $\mathrm{CD}^{+} 8^{+}$cells express Lyve-1 (Supplemental Figure S4). ${ }^{27-29}$


dominate in first-trimester human skin. A: Immunofluorescence triple staining for the indicated markers was performed on cryostat sections of prenatal and adult skin ( $n=3$ per group). Arrows denote $\mathrm{CD} 34^{-} \mathrm{CD} 144^{+}$Prox $1^{+}$, and arrowheads denote $\mathrm{CD} 34^{+} \mathrm{CD} 144^{+}$Prox $1^{-}$ vessels. B and C: Dot graphs depict the development of $\mathrm{CD} 144^{+}$vessel density and mean size. Each symbol represents one sample. Bars represent the mean of the investigated groups. D: Immunofluorescence triple labeling identifies CD144 ${ }^{+}$PAL-E ${ }^{-}$Lyve- $1^{+}$lymphatic (thick arrows), CD144 ${ }^{+}$PAL-E ${ }^{+}$Lyve- $1^{-}$venous and capillary (arrowheads), and CD144 ${ }^{+}$PAL-E ${ }^{-}$Lyve- $1^{-}$arterial (arrows) endothelial cells (ECs). First-trimester human skin occasionally has CD144 ${ }^{+} \mathrm{PAL}-\mathrm{E}^{+}$Lyve- $1^{+}$ vessels (double arrow). E: Dot graphs indicate the development of vessel phenotypes with advancing gestation. The percentages of CD144 ${ }^{+}$PAL-E ${ }^{+}$Lyve $^{-}$ veins and capillaries, $\mathrm{CD} 144^{+} \mathrm{PAL}-\mathrm{E}^{-}$Lyve- $1^{-}$arteries, CD144 ${ }^{+}$PAL-E ${ }^{-}$Lyve $-1^{+}$lymphatic vessels, and CD144 ${ }^{+}$PAL-E ${ }^{+}$Lyve- $1^{+}$vessels of total CD144 ${ }^{+}$vessels are given. Bars represent the mean of investigated groups. F: Immunofluorescence triple labeling identifies $\mathrm{CD}^{+} 1^{+}$PAL-E ${ }^{+}$Prox $1^{-}$blood vessels (arrowhead) and $\mathrm{CD} 31^{+} \mathrm{PAL}_{-} \mathrm{E}^{-}$Prox $1^{+}$lymphatic vessels (arrows). From 8 to 10 weeks of estimated gestational age, occasional $\mathrm{CD} 31^{+}$vessels with both PAL-E and Prox $1^{+}$are detected (double arrow). ${ }^{*} P<0.05,{ }^{* *} P<0.01$, and ${ }^{* * *} P<0.001$. Scale bars: $100 \mu \mathrm{m}(\mathbf{A}, \mathbf{D}$, and $\mathbf{F})$
With advancing gestational age, the distribution of vessels becomes increasingly similar to adult skin. Already after 11 weeks of EGA, CD144 ${ }^{+}$PAL-E $^{-}$vessels become more frequent, whereas CD144 ${ }^{+}$Lyve- $1^{+}$PAL- ${ }^{+}$vessels are less frequent (Figure 1, D and E). Overall, the phenotype of vessels in fetal skin (18 to 24 weeks of EGA) resembles the one observed in adult skin.

\section{Prox1, CD31, and CD144 Are the First LEC-Specific} Markers in Prenatal Human Skin

Our finding that in embryonic skin most CD144 ${ }^{+}$vessels are positive for the BEC marker PAL-E, the presence of few PAL- $\mathrm{E}^{+}$vessels expressing Prox 1 , and the scarcity of Lyve$1^{+}$LVs suggests that the cutaneous lymphatic system has 
A

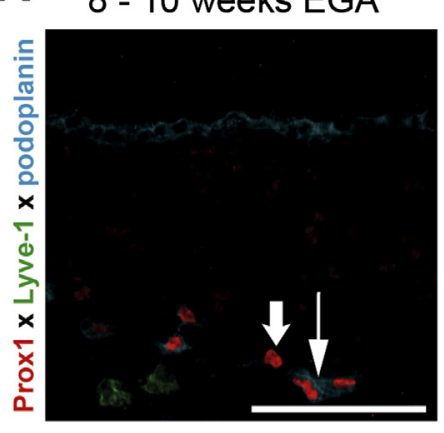

11 - 14 weeks EGA

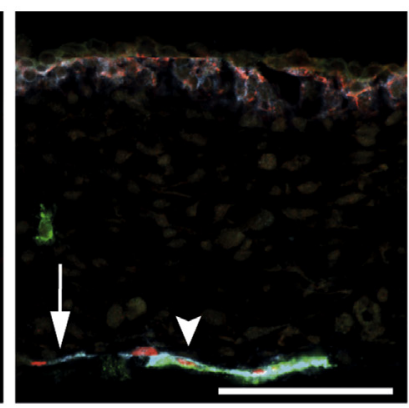

18 - 24 weeks EGA

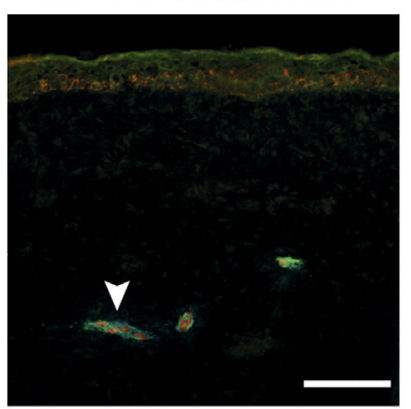

Adult

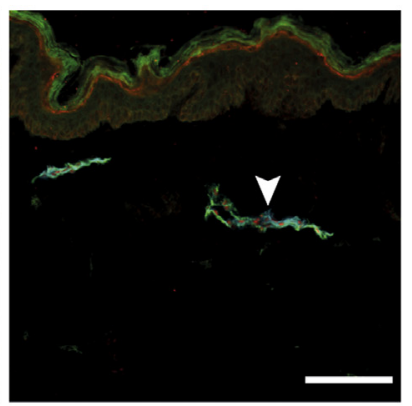

\section{B}

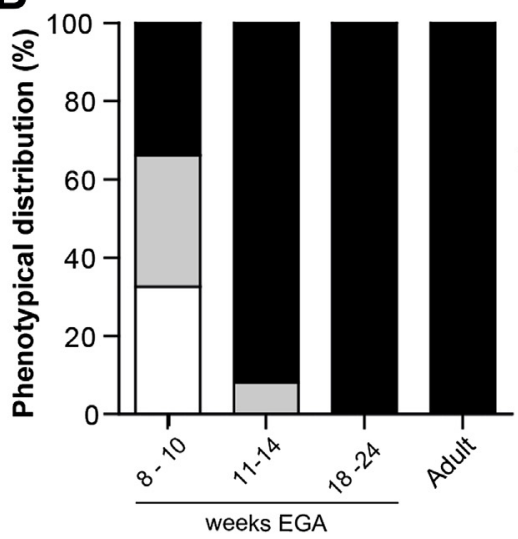

Prox $1^{+}$podoplanin ${ }^{-L y v e-1}{ }^{-}$

Prox ${ }^{+}$podoplanin ${ }^{+}$Lyve- ${ }^{-}$

Prox $1^{+}$podoplanin ${ }^{+}$Lyve $-1^{+}$
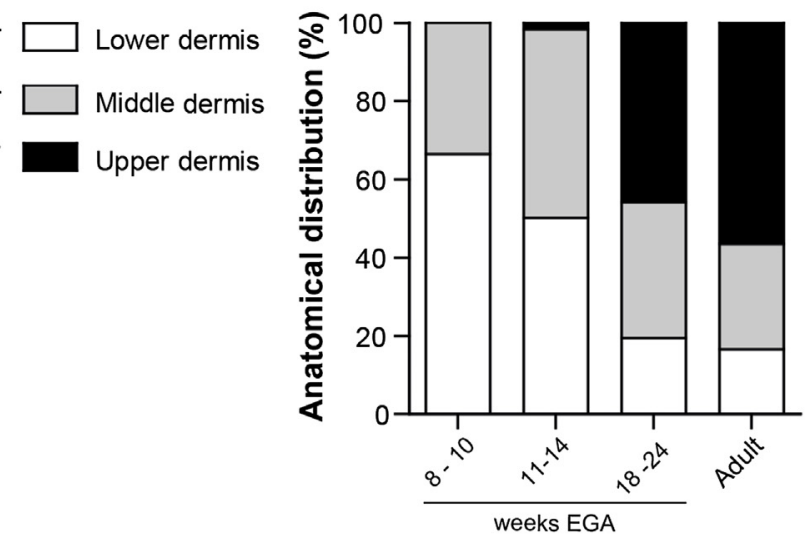

C 8-10 weeks EGA

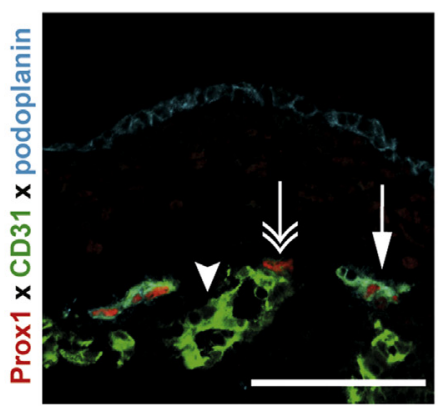

11 - 14 weeks EGA
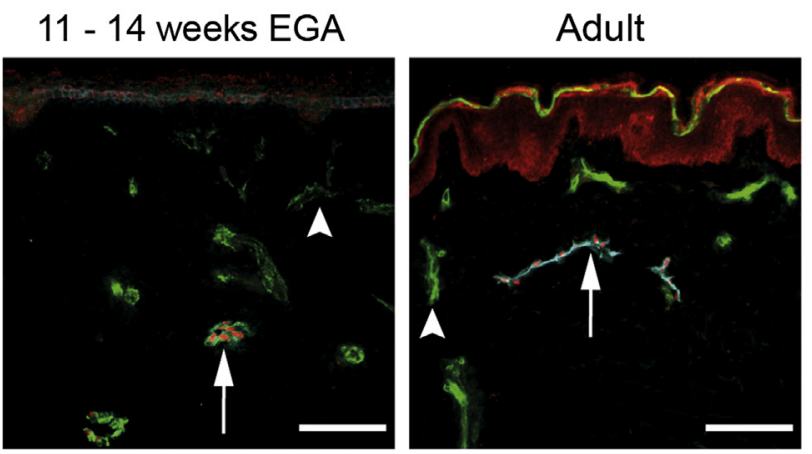

Figure 2 Lymphatic vessels develop in first-trimester human skin in a stepwise fashion. A: Immunofluorescence triple staining identifies Prox $1^{+}$Lyve$1^{-}$podoplanin ${ }^{-}$lymphatic endothelial cell progenitors (thick arrow) from 8 to 10 weeks of estimated gestational age (EGA). Apart from mature Prox $1^{+}$Lyve$1^{+}$podoplanin ${ }^{+}$lymphatic vessels (LVs) (arrowhead), Prox $1^{+}$Lyve-1 $1^{-}$podoplanin ${ }^{+}$(arrow) vessels are detectable in first-trimester human skin. B: Prox $1^{+}$Lyve- $1^{-}$podoplanin ${ }^{-}$LVs are detectable in 8 to 10 weeks of EGA and Prox $1^{+}$Lyve- $1^{-}$podoplanin ${ }^{+}$LVs only until 14 weeks of EGA. LVs accumulate in the lower parts of the dermis in the first trimester and acquire an adult-like distribution by midgestation. C: Immunofluorescence staining reveals that all Prox $1^{+}$nuclei express the pan-EC marker CD31. Both Prox $1^{+}$podoplanin ${ }^{-}$(double arrow) and Prox $1^{+}$podoplanin ${ }^{+}$(arrow) vessels express CD31. Arrowhead indicates CD31 ${ }^{+}$Prox $1^{-}$podoplanin ${ }^{-}$BVs. Scale bars: $100 \mu \mathrm{m}(\mathbf{A}$ and $\mathbf{C})$.

not yet fully and maturely evolved. ${ }^{3,6,33}$ To corroborate this notion and to investigate the temporal development of LVs in detail, we analyzed the expression of the LEC marker Prox 1 and podoplanin in relation to Lyve-1 using triple immunofluorescence. Unlike adult and fetal (18 to 24 weeks of EGA) skin that predominantly harbors Lyve $-1^{+} \operatorname{Prox} 1^{+}$podoplanin ${ }^{+}$ LVs (Figure 2, A and B, and Supplemental Figure S5), embryonic skin (8 to 14 weeks of EGA) has a marked heterogeneity. Only at 8 to 10 weeks of EGA, strong nuclear Prox 1 staining without concomitant staining for Lyve-1 or podoplanin is detectable $(32.6 \% \pm 33.1 \%, n=9)$ (Figure 2, $\mathrm{A}$ and
B, and Supplemental Figure S5). Although not expressing Lyve-1, one-third of Prox $1^{+}$nuclei are surrounded by podoplanin, which also stains consistently the basal keratinocyte layer throughout skin development $(33.5 \% \pm 29.4 \%, n=9)$ (Figure 2, $\mathrm{A}$ and $\mathrm{B}$, and Supplemental Figure S5). Remaining Prox $1^{+}$nuclei $(33.8 \% \pm 32.8 \%, n=9)$ (Figure 2B) are surrounded by Lyve-1 and podoplanin, thus revealing the mature LEC phenotype, yet being significantly less common than at 11 to 14 weeks of EGA $(91.9 \% \pm 11.5 \%, n=5, P=0.007)$ (Figure 2B). After 11 weeks of EGA, LVs that do not 

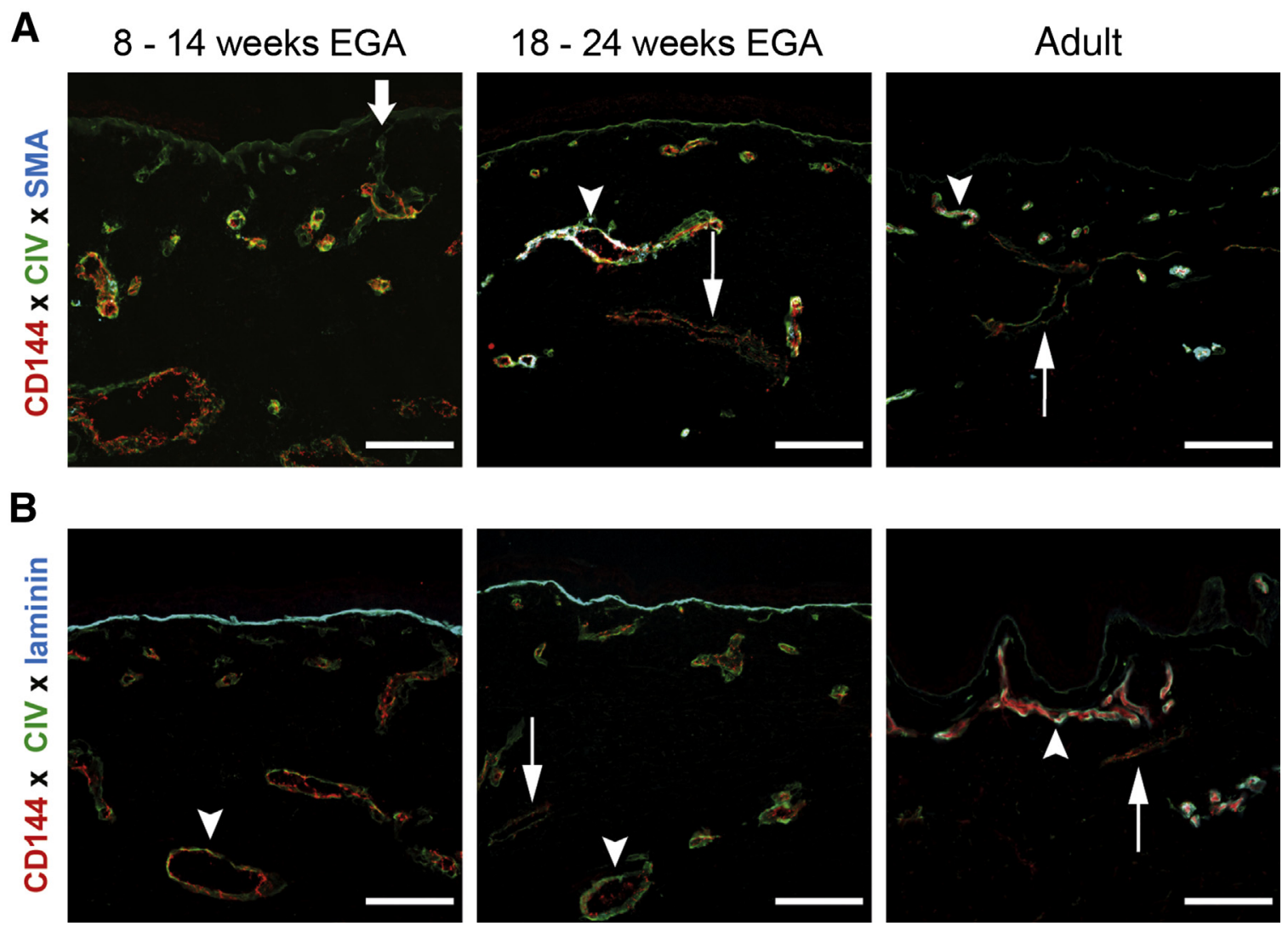

Figure 3 Tubes of collagen type IV (CIV) devoid of laminin, endothelial cells (ECs), or smooth muscle actin (SMA) $)^{+}$cells are identified beneath the epidermis in first-trimester human skin. A: Immunofluorescence labeling identifies CIV tubes devoid of CD144 ${ }^{+}$EC (thick arrow) and vessels mostly containing few SMA ${ }^{+}$cells in first-trimester human skin. Mature blood vessels contain SMA ${ }^{+}$cells and CIV (arrowhead), whereas mature lymphatic vessels contain no $\mathrm{SMA}^{+}$cells and less CIV (arrow). B: Laminin colocalizes with CIV in blood vessel (arrowhead) but not in lymphatic vessels (arrow) and reveals, apart from the dermoepidermal junction, a weaker staining intensity in prenatal skin. CIV tubes beneath the epidermis do not stain for laminin. Scale bars: $100 \mu \mathrm{m}$ (A and B).

express Lyve-1 are less frequent compared with earlier gestational time points $(8.15 \pm 11.5, n=5, P=0.09)$ (Figure 2, A and B) and are no longer detectable at midgestation. Of note, nuclei showing strong Prox 1 staining regardless of the expression of podoplanin are always surrounded by the pan-EC markers CD31 (Figure 2C and Supplemental Figure S6) and CD144 (Figure 1A), corroborating the notion that these Prox1expressing cells are indeed LECs or their respective precursors. In addition to their partly immature phenotype, LVs in the first trimester also have a different anatomical distribution than at midgestation or in adult life. They are generally not found in the upper third of the dermis and appear to accumulate in the lower parts of the dermis (Figure 2B). Although $1.6 \% \pm 2.2 \%(n=5)$ of all LVs at 11 to 14 weeks are found in the upper third of the dermis, this percentage increases to $45.8 \% \pm 28.7 \%(n=5$, $P=0.008)$ at midgestation, when no statistically significant difference in the distribution of LVs to adult skin is detected.

Taken together, it appears that LECs have a sequential order in the acquisition of their mature phenotype: $\mathrm{CD} 31^{+} \mathrm{CD} 144^{+}$Prox $1^{+}$cells partly lose co-expression of the venous EC marker PAL-E until the end of the first trimester and concomitantly acquire first podoplanin and then Lyve-1.

\section{Expression of SMA and Laminin Is Rare around Vessels in First-Trimester Skin}

The maturation of the developing vascular system involves the deposition of a specific basement membrane and, depending on the vessel type, the recruitment of $\mathrm{SMA}^{+}$mural cells, eventually leading to the stabilization of the newly formed vessels. ${ }^{4,34}$ Triple immunofluorescence staining with CD144, CIV, and SMA antibodies confirmed that in healthy adult skin mature arteries and veins are composed of a luminal layer of ECs surrounded by a continuous basement membrane composed of CIV and contractile cells containing SMA (Figure $3 \mathrm{~A}$ and Supplemental Figure S7). By contrast, most lymphatic capillaries and vessels do not contain $\mathrm{SMA}^{+}$cells and are characterized by a discontinuous basement membrane expressing lower amounts of CIV (Figure 3A and Supplemental Figure S7).

To infer about the temporal development of these structural vessel components, we found that most $\mathrm{CD} 144^{+} \mathrm{ECs}$ are surrounded by CIV at 8 to 14 weeks of EGA. Intriguingly, the previously described small band underneath the primitive epidermis devoid of $\mathrm{CD}_{144^{+}}$or $\mathrm{CD} 34^{+}$ECs contains 


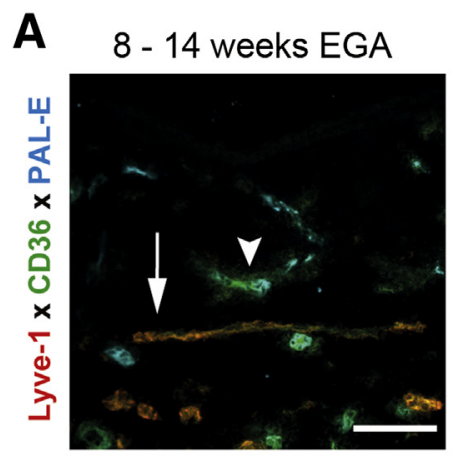

18 - 24 weeks EGA

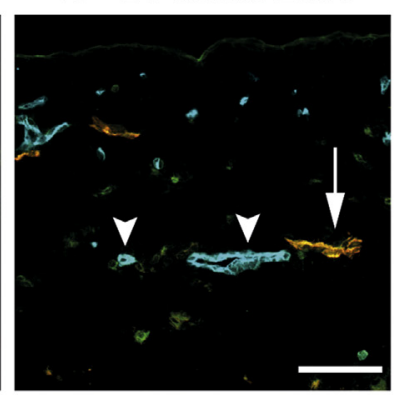

C



E
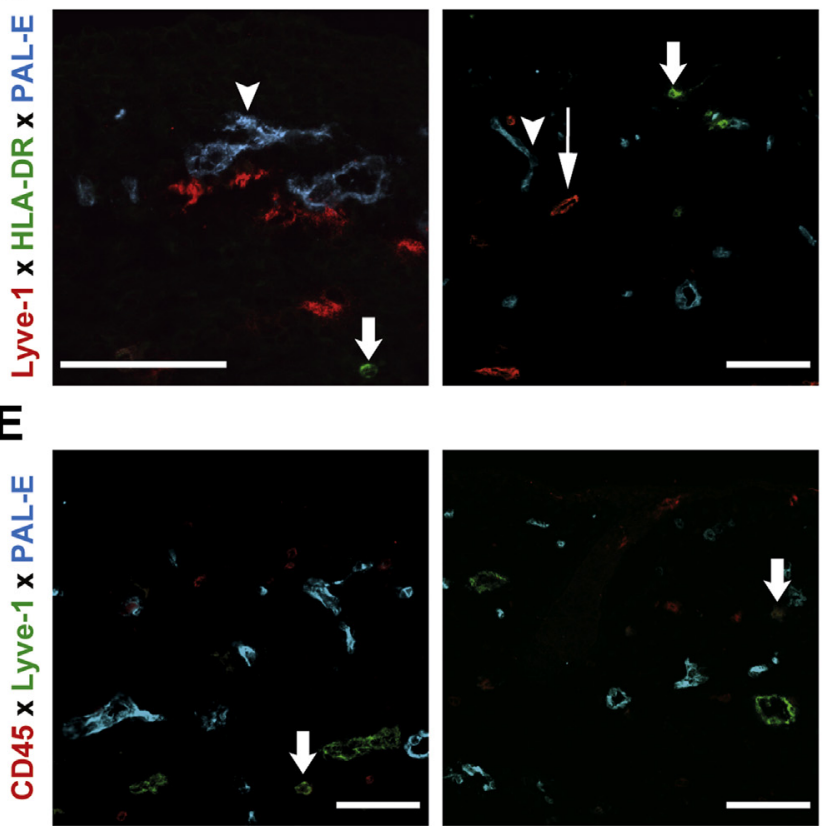

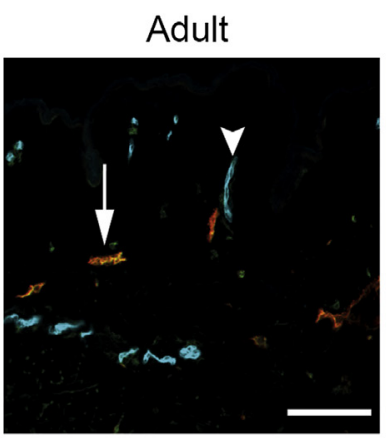

B

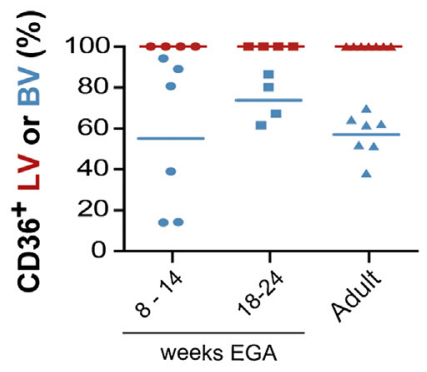

D
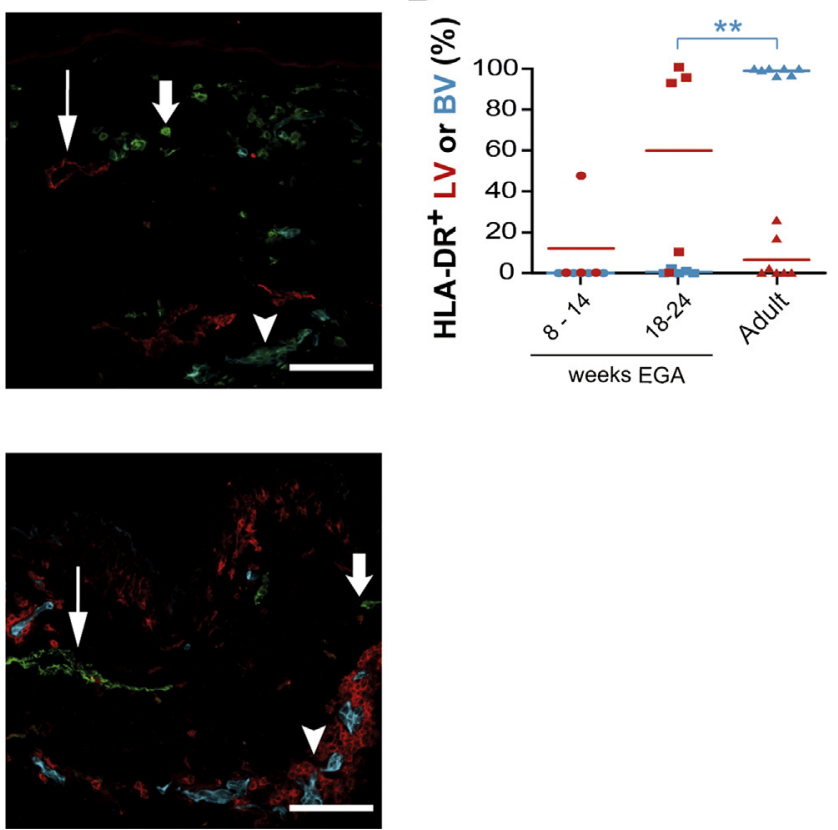

Figure 4 Analysis of accessory molecules on blood and lymphatic vessels. A: CD36 is expressed on lymphatic vessels (LVs) (arrow) and on a subset of blood vessels (BVs) (arrowhead) throughout development. B: Dot graphs indicate the expression of CD36 on BVs (blue) and LVs (red) with advancing gestation. The percentage of $\mathrm{CD} 36^{+} \mathrm{PAL}-\mathrm{E}^{+}$BVs (blue) and CD36 ${ }^{+} \mathrm{Lyve}-1^{+} \mathrm{LVs}$ (red) relative to all PAL-E $\mathrm{E}^{+}$or Lyve- $1^{+}$positive vessels. Bars represent the mean of investigated groups. C: HLA-DR is not found on BVs (arrowhead) but on some LVs (arrow). Thick arrow indicates HLA-DR ${ }^{\text {high }}$ antigen-presenting cells. D: Dot graphs indicate the expression of HLA-DR on BVs (blue) and LVs (red) with advancing gestation. The percentage of HLA-DR ${ }^{+}$PAL-E ${ }^{+}$BVs (blue) and HLA-DR ${ }^{+}$Lyve- $1^{+}$ LVs (red) relative to all PAL-E- or Lyve-1-positive vessels. HLA-DR expression on endothelial cells (ECs) between prenatal and adult skin differs significantly. Each symbol represents one sample. Bars represent the mean of investigated groups. E: CD45 leukocytes are sparsely found in prenatal skin but are clustered around PAL-E ${ }^{+}$vessels in adult skin (arrowhead). No accumulation of leukocytes is observed around LVs (arrow). Lyve- $1^{+}$macrophages (thick arrow) are identified in all developmental stages analyzed ( $n=5$ to 7 for all staining and age groups investigated). ${ }^{* *} P<0.01$ (D). Scale bars: $100 \mu \mathrm{m}$.

tube-like structures composed of CIV. ${ }^{19}$ Occasionally, these structures appear to extend from deeper vascular structures that contain $\mathrm{CD} 144^{+}$ECs (Figure 3A), suggesting that angiogenesis during skin development involves the initial formation of tracks made of basement membrane components followed by their colonization with ECs. Only a few vessels contain SMA, and the staining is generally punctate and much weaker than in adult skin (Figure 3A). With advancing gestation, the staining pattern in fetal skin (18 to 24 weeks of EGA) resembles the one in adult skin regarding higher expression levels of CIV in BVs than in LVs (Figure 3A) and the staining intensity of SMA. The subepidermal band devoid of CD144 ${ }^{+}$ECs during the first trimester now contains ECs surrounded by CIV, although occasionally some CIV tubes in fetal and adult skin do not contain ECs, perhaps representing cutting artifacts. We next examined whether these CIV tubes contain laminin, another central component of the basement membrane. We found that in prenatal skin laminin is expressed most strongly in the dermoepidermal junctions, whereas the basement membrane of CD144 ${ }^{+}$ vessels has weak or no expression. Except from branching areas, CIV tubes devoid of CD144 ${ }^{+}$EC underneath the epidermis do not express laminin. Similar to the expression of SMA, the intensity of laminin expression increases with gestational age (Figure 3B and Supplemental Figure S8). However, the adult pattern (ie, a stronger staining of the vascular basement membranes of BVs than the dermoepidermal junction) is not reached by the end of the second trimester. Similar to adult skin, LVs in prenatal skin do not express laminin in their basement membrane (Figure 3B). 
Taken together, the vessel walls in first-trimester human skin appear to be immature and thus possibly functionally compromised when compared with vessels in fetal skin and adult skin given their lower content of $\mathrm{SMA}^{+}$mural cells and laminin as well as the uniform CIV expression.

\section{CD36 Is Expressed on LVs throughout Intrauterine Skin Development}

In prenatal and adult skin, a subset of ECs expresses CD36, ${ }^{13,18,35}$ and LECs in adult skin express much higher levels of CD36 than BECs. ${ }^{12}$ Given the abundance of CD36 expression in developing human skin, ${ }^{18}$ we analyzed the sequential CD36 expression during ontogeny. We found an identical staining pattern between prenatal and adult skin, namely, that all Lyve- $1^{+}$LVs (Figure $4, \mathrm{~A}$ and B, and Supplemental Figure S9) and Lyve- $1^{+}$macrophages express CD36 and that a subset of PAL- ${ }^{+}$vessels in fetal and adult skin (Figure 4, A and B) stained for CD36. Given the diverse functions of $\mathrm{CD} 36$, including its role as a receptor for collagen, the early presence of this molecule might hint at the role of CD36 in the EC-matrix interaction involved in angiogenesis.

\section{Some LECs but Not BECs Express HLA-DR in Prenatal Human Skin}

In healthy adult skin, HLA-DR is constitutively expressed by BECs but not by LECs. ${ }^{12,14,36}$ Recently, we found that in prenatal skin the occasional expression of HLA-DR on ECs is not acquired before 18 to 24 weeks of EGA, ${ }^{11}$ but it remains unknown whether LECs and/or BECs express HLA-DR during skin development. We found that in prenatal skin throughout development PAL-E never colocalizes with HLADR (Figure 4, C and D, and Supplemental Figure S10), whereas in five of nine samples investigated, predominantly at 18 to 24 weeks of EGA, Lyve- ${ }^{+}$LVs weakly express HLADR (Figure 4C). As previously described, ${ }^{11}$ HLA-DR $^{+}$leukocytes are sparsely scattered in both the epidermis and dermis, and of these, only a small subset express Lyve- 1 . This staining pattern in developing skin is in stark contrast to adult skin, which has co-expression of HLA-DR with PAL-E (Figure 4C and Supplemental Figure S10) and clustering of HLA-DR ${ }^{+}$leukocytes around PAL-E ${ }^{+}$BVs. Lyve- $1^{+}$LVs in most samples investigated do not express HLA-DR (Figure 4C and Supplemental Figure S10), and if they do, they only have faint expression. The differential expression of HLA-DR on vessels in fetal skin (ie, its absence on PAL-E ${ }^{+}$ and its partial presence on Lyve- $1^{+}$vessels) compared with adult skin could point at temporary developmental steps in the acquisition of the cutaneous immune system.

\section{Cutaneous Leukocytes in Prenatal Human Skin Are Not Clustered around BVs as in Adult Skin}

We next assessed the distribution of $\mathrm{CD}_{4} 5^{+}$leukocytes relative to BVs and LVs in prenatal and adult skin. In adult
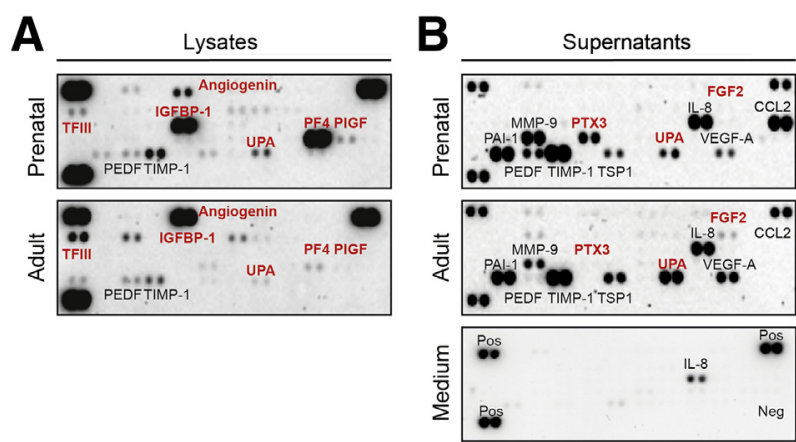

Figure 5 Fetal and adult skin lysates and culture supernatants have a complex profile of angiogenic factors. Lysates of prenatal (11 to 24 weeks, $n=7$ ) and adult skin (44 to 51 years, $n=4)($ A) and supernatants of single-cell suspensions of prenatal (11 to 24 weeks of estimated gestational age, $n=8$ ) and adult (19 to 46 years, $n=3$ ) skin (B) were analyzed for the molecular pattern of angiogenic factors using commercially available protein arrays. Proteins with statistically significant differences are highlighted in red. Neg, negative; Pos, positive.

skin, $\mathrm{CD} 45^{+}$cells are located in the more superficial parts of the dermis around $\mathrm{PAL}^{+} \mathrm{E}^{+} \mathrm{BVs}$ (Figure $4 \mathrm{E}$ and Supplemental Figure S11) and less frequently interstitially but not around Lyve- $1^{+}$LVs (Figure 4E) ${ }^{28}$ In contrast, leukocytes in prenatal human skin are significantly less frequent $^{11}$ and appear to be randomly distributed without any specific association with BVs or LVs. CD45 ${ }^{+}$cells are not detectable inside LVs in prenatal or adult human skin; however, they are occasionally found at the luminal side of BVs in first-trimester human skin (data not shown). Interestingly, some Lyve- $1^{+}$mononuclear cells co-express CD45 (Figure 4E), representing a subset of skin macrophages. $^{27-29}$ Taken together, even though cutaneous LVs and BVs in the second trimester are present and phenotypically mature, they lack the ample perivascular cover of leukocytes seen in adult skin.

\section{Molecular Profile of Angiogenic Molecules Differs in Lysates and Culture Supernatants of Prenatal and Adult Human Skin}

On establishment of the first vessels, the vascular system expands by angiogenesis (ie, the growth of new BVs from existing ones). ${ }^{5,6}$ This process requires a multitude of angiogenic proteins and their corresponding receptors, celladhesion proteins, matrix components, and transcription factors orchestrating the development of BVs and LVs. ${ }^{37}$ To examine factors responsible for the development of BVs and LVs in prenatal human skin, we comparatively assessed the molecular pattern of skin lysates and supernatants derived from cultured whole skin single-cell suspensions of fetal and adult skin using protein microarrays. We found that prenatal skin possesses a complex expression pattern of proangiogenic and antiangiogenic proteins (Figure 5A and Supplemental Table S1). Although some proangiogenic molecules are found in higher levels than in adult skin 




Figure 6 Scheme of cutaneous blood and lymphatic vessel (LV) development. The vascular network at the earliest developmental stages is tilted toward a venous vessel phenotype and only gradually develops the phenotypes reminiscent of adult arteries and LVs (upper panel). Lymphatic progenitor cells at 8 weeks of estimated gestational age (EGA) express CD31, CD144, Prox1, and temporary PAL-E (lower panel). At that developmental stage, not all lymphatic progenitor cells express podoplanin or Lyve-1, which are acquired with advancing gestational age in a stepwise fashion. The developmental time scale is shown along the bottom of the figure. ECs, endothelial cells; LVs, lymphatic vessels.

[eg, PIGF (12-fold), vascular endothelial growth factor (VEGF)-C (ninefold)], other proteins believed to be crucial for angiogenesis, such as VEGF or angiopoetins, had no significant difference. Intriguingly, some proangiogenic factors and regulatory proteins are found at higher levels in adult skin [eg, IGFBP-1 (43-fold), angiogenin (sevenfold), UPA (fourfold), tissue factor III (threefold),] whereas the antiangiogenic protein platelet factor 4 is found in 30-fold higher levels in prenatal skin. Surprisingly, the secretion pattern of several proangiogenic and antiangiogenic proteins of prenatal and adult skin is similar with detectable levels of IL-8, TIMP-1, TSP1, VEGF, MMP-9, and PAI-1 (Figure 5B and Supplemental Table S2). Of note, CCL2 (80-fold) and PTX3 (sevenfold) were found in higher, whereas FGF-2 (fivefold) and UPA (fourfold) were found in lower intensities in supernatants of prenatal than in adult supernatants. Together, these data suggest an unexpected, intricate regulation of angiogenesis during ontogeny.

\section{Discussion}

This study provides a detailed analysis of the development of the prenatal human cutaneous blood and lymphatic vascular system. We found that the vascular network at the earliest developmental stages investigated in this study is tilted toward a venous vessel phenotype and only gradually develops the phenotypes reminiscent of adult arteries and LVs (Figure 6). However, the phenotypic repertoire of both BVs and LVs in second-trimester human skin essentially resembles the one in adult skin, with the exceptions of the short-term, ectopic expression of HLA-DR on LVs, less laminin expression in the basement membrane of BVs, and the marked absence of leukocytes accumulating in the superficial parts of the dermis and around BVs.
The Typical Phenotypic Hallmarks of Veins, Arteries, and LVs Develop during the First Trimester in Prenatal Human Skin

In human embryonic skin, BVs have been described as early as 6 weeks of EGA. ${ }^{15}$ We identified $\mathrm{CD} 34^{+} \mathrm{CD} 144^{+}$Prox $1{ }^{-}$ $\mathrm{BVs}$ as the major and $\mathrm{CD} 34^{-/ \text {weak }} \mathrm{CD} 144^{+} \mathrm{Prox} 1^{+} \mathrm{LVs}$ as a minor vessel phenotype in first-trimester human skin, the latter increasing in frequency as the pregnancy advances. Compared with adult skin, prenatal skin at all time points of investigation has a significantly higher vessel density. At 8 to 10 weeks of EGA, the vessel size is significantly smaller compared with those in adults but already at 11 weeks reaches adult size. ${ }^{26}$ It is likely that this higher vascularity accounts for the higher nutrient requirements of the growing skin. Alternatively, it is possible that, similar to hair follicles, bigger, already formed vessels move apart after birth proportionally to the growth of the body and the skin. ${ }^{38}$

We were surprised that most $\mathrm{CD} 144^{+}$vessels until 10 weeks of EGA express PAL-E. Given that in some samples only CD $144^{+}$PAL-E ${ }^{+}$venous vessels and, consequently, no CD $144^{+}$PAL-E $^{-}$arterial vessels are detectable, we assume that, at least phenotypically, the specification of arteries and veins has not occurred. This assumption is supported by our observation that virtually all $\mathrm{CD} 144^{+}$vessels lack $\mathrm{SMA}^{+}$ mural cells characteristic for arteries and could be verified by studying the expression of the venous and arterial fate cell marker ephrinB2 or EphB4, respectively, in firsttrimester human skin. ${ }^{39}$ The first arterial CD144 ${ }^{+}$PAL-E ${ }^{-}$ vessels covered by a dense layer of SMA are detectable around the end of the first trimester, and numerous, phenotypically mature BVs are eventually found in the second trimester.

\section{Vessels in First Trimester Human Skin Have Fewer $\mathrm{SMA}^{+}$Mural Cells than in Adult Skin}

It is currently believed that vascular stability and, consequently, optimal functionality seen in vessels of adult, healthy skin requires the integration of $\mathrm{SMA}^{+}$mural cells (ie, pericytes and smooth muscles cells) into the vessel wall and the deposition of all relevant structural components into the basement membrane. ${ }^{34}$ Our observation that $\mathrm{SMA}^{+}$cells in first-trimester human skin are present in some vessels only and generally have lower expression levels suggests that these vessels possess a compromised function compared with vessels in fetal and adult skin. Consequently, it is possible that they are not able to regulate perfusion and are more fragile and leaky compared with vessels in later developmental stages. According to murine models, $\mathrm{SMA}^{+}$cells are recruited from the undifferentiated mesenchyme surrounding the developing vessels; however, the precise nature and origin of $\mathrm{SMA}^{+}$cells remains to be determined. ${ }^{34}$ The process of pericyte and $\mathrm{SMA}^{+}$cell recruitment critically depends on the release of platelet-derived growth factor- $\beta$, transforming growth factor- $\beta$, and angiopoietin-1 from ECs. ${ }^{40-42}$ Intriguingly, our data do 
not reveal higher levels of these proteins or their precursors in prenatal skin. However, this does not preclude a functional role given that these factors act in an autocrine or paracrine manner and may thus not be secreted. CIV, one central component of the vascular basement membrane and another crucial component for vascular stability, is readily detectable at 8 weeks of EGA. Interestingly, in contrast to adult and secondtrimester skin, for which differential expression of CIV allows the gross separation of BVs and LVs, the staining intensity of CIV is similar in all vessels during the first trimester. Accordingly, the expression of CIV around nascent LVs has to decrease at the end of the first trimester. This finding is consistent with observations in murine models that reveal a reduction of CIV and laminin in the budding LECs. ${ }^{3,31}$

\section{Subepidermal Tubes Composed of CIV and Devoid of ECs May Act as Scaffolds for Rapid Revascularization Early during Development}

A previous study has described a subepidermal band devoid of $\mathrm{CD} 34^{+}$cells underneath the epidermis. ${ }^{19}$ We confirmed that below the epidermis no $\mathrm{CD} 31^{+}, \mathrm{CD} 34^{+}$, or $\mathrm{CD} 144^{+}$ ECs are present in first-trimester human skin but that tubelike structures made of CIV that lack laminin or $\mathrm{SMA}^{+}$cells are found within this space. These abundant CIV tubes extending from deeper vessels and occasionally reaching the dermoepidermal junction appear as empty sleeves. At this time, papillary tips between elongated rete ridges have not yet formed. One could postulate that they were created by sprouting and subsequent retracting vascular sprouts, a phenomenon that is well documented in the developing zebra fish vasculature. ${ }^{43}$ These empty sleeves may then act as scaffolds for the formation of capillary loops once papillary tips and rete ridges have been properly formed. Of note, empty sleeves are also found in tumors undergoing antiangiogenic treatment. After cessation of the treatment, these sleeves provide a scaffold for rapid revascularization and are a reservoir of angiogenic factors, including VEGF. $^{44,45}$

\section{During Ontogeny, LVs Gradually Develop Their Phenotype}

During development, the lymphatic vasculature appears after the formation of the blood vascular system and then develops in parallel. ${ }^{3,6}$ To date, murine models favor the venous origin of LECs in the anterior cardinal vein over the direct development from mesenchymal cells. Regardless of the origin, murine LECs then progress in a stepwise manner, eventually forming a phenotypically mature lymphatic network only shortly before birth. ${ }^{3}$ We found that at approximately 8 weeks of EGA human developing skin contains Prox $1^{+}$cells expressing the pan-EC markers CD31 and CD144 and the venous EC marker PAL-E but testing negative for CD34, Lyve-1, and podoplanin. This phenotype is consistent with that of LEC precursors, given that Prox 1 probably serves as the master switch for the lymphatic phenotype in mice and humans. ${ }^{3,31,46,47}$ The detection of Prox $1^{+}$PAL- $E^{\text {weak }}$ and Lyve- $1^{+}$PAL- ${ }^{\text {weak }}$ vessels in firsttrimester human skin might hint at the venous origin of LEC, a concept that has been found in mice. ${ }^{31,47}$ Of note, co-expression of Lyve-1 and PAL-E has also been described in cutaneous diseases, such as psoriasis or eczema. It has been suggested that a low-level expression of the lymphatic marker Lyve-1 on BECs reflects EC activation rather than transdifferentiation of BECs into LECs. ${ }^{30}$

Already during the first trimester, the phenotype of LECs changes from Prox $1^{+}$Lyve- $1^{-}$podoplanin ${ }^{-}$to Prox $1^{+}$Lyve$1^{+}$podoplanin ${ }^{+}$via Prox $1^{+}$Lyve- $1^{-}$podoplanin ${ }^{+}$. At that developmental stage, LVs are primarily found in the lower and middle third of the developing dermis, developing the spatial distribution reminiscent of adult skin already by the second trimester. The LEC-specific tyrosine kinase VEGFR3 has been identified in human skin at 11 weeks of $\mathrm{EGA}^{23,24}$; however, its temporal development in humans is currently not known. Given the early expression of VEGFR3 by both blood and lymphatic ECs during early development, ${ }^{7,24}$ it seems probable that it is already found on Prox $1^{+}$Lyve- $1^{-}$podoplanin ${ }^{-}$LEC precursors. Similar to comparable stages of murine development, ${ }^{31}$ the phenotypic maturation of LVs corresponds with the progressive decrease of the basement membrane component CIV during the second trimester. Taken together, in humans the development of the mature LV phenotype is a stepwise process that requires several months, only slightly differing in the sequential order of marker acquisition compared with mice, and is almost completed during the second trimester. It can be speculated that similar to mice in humans these processes are driven by VEGF-C as well, given its higher levels in prenatal skin. ${ }^{48}$ However, although phenotypically mature, it is unknown whether antigen-presenting cells home draining lymph nodes via functional LVs in the second trimester. Although we did not detect $\mathrm{CD} 45^{+}, \mathrm{CD} 36^{+}$, or

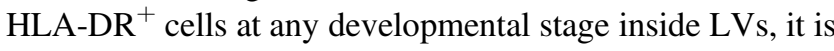
conceivable that short transit times or technical issues impede their detection. ${ }^{28}$ Investigating LVs in preterm deliveries with fetal dermatitis after microbial invasion of the amniotic fluid or analyzing the secretion of chemokines known to regulate leukocyte migration such as CCL21 should help to assess this important issue..$^{31,49}$

\section{Second-Trimester Cutaneous Vessels Contain Fewer Perivascular Leukocytes than Adult Skin}

CD45 ${ }^{+}$cells in adult skin are found in significant numbers both interstitially and around BVs. Although dendritic cells and macrophages are found at both locations, $\mathrm{T}$ cells are predominantly perivascular. ${ }^{28}$ Our observation that the few leukocytes in prenatal human skin appear to be randomly distributed without any specific association with BVs or LVs can be explained by low numbers of leukocytes in developing skin and the lack of skin-homing $\mathrm{T}$ cells in 
human fetal skin. ${ }^{21}$ It is likely that fetal leukocytes use the same molecular mechanisms for extravasation as adult ones, suggested by the robust expression of the adhesion molecules CD62P and CD54 on ECs in the second-trimester skin. ${ }^{19}$ Clinical evidence further supports the notion that this primitive fetal system of leukocyte diapedesis is functional, exemplified by the massive influx of lymphocytes, macrophages, and granulocytes in fetal dermatitis associated with microbial invasion of the amniotic fluid. ${ }^{49}$

The function of other accessory molecules on ECs is less clear. We were surprised to detect expression of HLA-DR on LECs of some samples but not on BECs, thus contrasting adult skin with exclusive and constitutive expression of HLA-DR on BECs. The function of HLA-DR-expressing LECs during midgestation is yet unknown, and because of its short-term expression, one may speculate that they have regulatory functions for the nascent immune system. ${ }^{11} \mathrm{By}$ contrast, expression of CD36 on BVs and LVs in prenatal human skin is similar to adult skin and has been found in the earliest samples investigated.

Apart from its expression on LVs, Lyve-1 is also found at low expression levels on some FXIIIa ${ }^{+} \mathrm{CD} 11 \mathrm{c}^{-}$macrophages in adult skin. ${ }^{27-29} \mathrm{We}$ found that these Lyve- $1^{+}$cells in adult skin are uniformly $\mathrm{CD} 36^{+}, \mathrm{CD}^{+} 8^{+}$, and $\mathrm{CD} 163^{+}$but heterogeneous with regard to the expression of HLA-DR and CD45. In embryonic skin these Lyve- $1^{+}$single cells appear to be more frequent than in adult skin but exhibit the identical marker profile. It remains to be investigated whether these macrophages with their proximity to vessels produce angiogenic factors or are able to transdifferentiate into LECs. ${ }^{46,50}$

\section{Prenatal Human Skin Does Not Reveal the Expected Bent to Proangiogenic Molecules}

The regulation of angiogenesis depends on a fine balance between proangiogenic and antiangiogenic factors and involves substantial tissue remodeling. ${ }^{37}$ Because of the rapid growth and the resulting nutrient requirements, it can be inferred that during skin development proangiogenic factors outnumber antiangiogenic factors. The analysis of skin lysates and cell culture supernatants of prenatal and adult skin for angiogenic factors revealed a complex, rather unexpected molecular profile of different levels of proangiogenic (IL-8, VEGF-A, VEGF-C, PlGF, angiogenin), antiangiogenic (TSP1, platelet factor 4), and various regulatory (TIMP, MMP9, PAI-1, UPA, IGFBP-1) proteins. Taken together, we were surprised to discover a cytokine milieu without the expected bent to proangiogenic molecules in prenatal human skin. Possible explanations for this finding could be that angiogenic factors in prenatal human skin act in an autocrine or paracrine manner and are thus not secreted. The contribution of other, so far unidentified molecules is possible or, less likely, developmental programs independent from angiogenic factors. Of note, various physiological and pathological conditions including wound healing, vascular malformations, and tumor angiogenesis, events which might have taken place during ontogeny, might be recapitulated. ${ }^{39,51}$
Thus, a better understanding of the physiologic development of BVs and LVs during development should allow us to better comprehend and treat pathologic conditions with a derailed angiogenesis.

\section{Conclusion}

In summary, this study provides enticing new insights into the development and phenotypic characteristics of BECs and LECs in human prenatal skin. Given the similarities between developmental and tumor angiogenesis, it is possible that the comparison of the molecular pattern of angiogenic pathways in both circumstances could reveal new molecules involved in angiogenesis, eventually perhaps even leading to the development of new therapeutic avenues.

\section{Acknowledgments}

C.S., M.M., A.B., L.N., R.R., and L.B. performed the experiments and analyzed the data; C.F. and W.E. provided study material; C.S., W.B., P.P., and A.E.-B. conceived and designed the research; and C.S. and A.E.-B. wrote the manuscript.

\section{Supplemental Data}

Supplemental material for this article can be found at http://dx.doi.org/10.1016/j.ajpath.2015.05.006.

\section{References}

1. Kriehuber E, Breiteneder-Geleff S, Groeger M, Soleiman A, Schoppmann SF, Stingl G, Kerjaschki D, Maurer D: Isolation and characterization of dermal lymphatic and blood endothelial cells reveal stable and functionally specialized cell lineages. J Exp Med 2001, 194:797-808

2. Huggenberger R, Detmar M: The cutaneous vascular system in chronic skin inflammation. J Investig Dermatol Symp Proc 2011, 15:24-32

3. Oliver G: Lymphatic vasculature development. Nat Rev Immunol 2004, 4:35-45

4. Breier G: Angiogenesis in embryonic development - a review. Placenta 2000, 21(Suppl A):S11-S15

5. Carmeliet P: Angiogenesis in life, disease and medicine. Nature 2005, 438:932-936

6. Alitalo K, Tammela T, Petrova TV: Lymphangiogenesis in development and human disease. Nature 2005, 438:946-953

7. Choi I, Lee S, Hong YK: The new era of the lymphatic system: no longer secondary to the blood vascular system. Cold Spring Harb Perspect Med 2012, 2:a006445

8. Ordonez NG: Immunohistochemical endothelial markers: a review. Adv Anat Pathol 2012, 19:281-295

9. Xu B, deWaal RM, Mor-Vaknin N, Hibbard C, Markovitz DM, Kahn ML: The endothelial cell-specific antibody PAL-E identifies a secreted form of vimentin in the blood vasculature. Mol Cell Biol 2004, 24:9198-9206

10. Keuschnigg J, Tvorogov D, Elima K, Salmi M, Alitalo K, Salminen T, Jalkanen S: PV-1 is recognized by the PAL-E antibody and forms complexes with NRP-1. Blood 2012, 120:232-235

11. Schuster C, Vaculik C, Fiala C, Meindl S, Brandt O, Imhof M, Stingl G, Eppel W, Elbe-Bürger A: HLA-DR ${ }^{+}$leukocytes acquire CD1 antigens in embryonic and fetal human skin and contain functional antigen-presenting cells. J Exp Med 2009, 206:169-181 
12. Amatschek S, Kriehuber E, Bauer W, Reininger B, Meraner P, Wolpl A, Schweifer N, Haslinger C, Stingl G, Maurer D: Blood and lymphatic endothelial cell-specific differentiation programs are stringently controlled by the tissue environment. Blood 2007, 109:4777-4785

13. Petzelbauer P, Bender JR, Wilson J, Pober JS: Heterogeneity of dermal microvascular endothelial cell antigen expression and cytokine responsiveness in situ and in cell culture. J Immunol 1993, 151:5062-5072

14. Angel CE, George E, Ostrovsky LL, Dunbar PR: Comprehensive analysis of MHC-II expression in healthy human skin. Immunol Cell Biol 2007, 85:363-369

15. Johnson CL, Holbrook KA: Development of human embryonic and fetal dermal vasculature. J Invest Dermatol 1989, 93:10S-17S

16. Fine JD, Smith LT, Holbrook KA, Katz SI: The appearance of four basement membrane zone antigens in developing human fetal skin. J Invest Dermatol 1984, 83:66-69

17. Tonnesen MG, Jenkins D Jr, Siegal SL, Lee LA, Huff JC, Clark RA: Expression of fibronectin, laminin, and factor VIII-related antigen during development of the human cutaneous microvasculature. J Invest Dermatol 1985, 85:564-568

18. Schuster C, Mildner M, Mairhofer M, Bauer W, Fiala C, Prior M, Eppel W, Kolbus A, Tschachler E, Stingl G, Elbe-Bürger A: Human embryonic epidermis contains a diverse Langerhans cell precursor pool. Development 2014, 141:807-815

19. Davies JR, Dyson M, Mustafa Y, Compton F, Perry ME: The ontogeny of adhesion molecules expressed on the vascular endothelium of the developing human skin. J Anat 1996, 189(Pt 2):373-382

20. Karelina TV, Goldberg GI, Eisen AZ: Matrix metalloproteinases in blood vessel development in human fetal skin and in cutaneous tumors. J Invest Dermatol 1995, 105:411-417

21. Schuster C, Vaculik C, Prior M, Fiala C, Mildner M, Eppel W, Stingl G, Elbe-Bürger A: Phenotypic characterization of leukocytes in prenatal human dermis. J Invest Dermatol 2012, 132:2581-2592

22. Fleming JE Jr, Haynesworth SE, Cassiede P, Baber MA, Caplan AI: Monoclonal antibody against adult marrow-derived mesenchymal stem cells recognizes developing vasculature in embryonic human skin. Dev Dyn 1998, 212:119-132

23. von Kaisenberg CS, Wilting J, Dork T, Nicolaides KH, MeinholdHeerlein I, Hillemanns P, Brand-Saberi B: Lymphatic capillary hypoplasia in the skin of fetuses with increased nuchal translucency and Turner's syndrome: comparison with trisomies and controls. Mol Hum Reprod 2010, 16:778-789

24. Lymboussaki A, Partanen TA, Olofsson B, Thomas-Crusells J, Fletcher CD, de Waal RM, Kaipainen A, Alitalo K: Expression of the vascular endothelial growth factor $\mathrm{C}$ receptor VEGFR-3 in lymphatic endothelium of the skin and in vascular tumors. Am J Pathol 1998, 153:395-403

25. Ecker RC, Steiner GE: Microscopy-based multicolor tissue cytometry at the single-cell level. Cytometry A 2004, 59:182-190

26. Gunin AG, Petrov VV, Golubtzova NN, Vasilieva OV, Kornilova NK: Age-related changes in angiogenesis in human dermis. Exp Gerontol 2014, 55:143-151

27. Attout T, Hoerauf A, Denece G, Debrah AY, Marfo-Debrekyei Y, Boussinesq M, Wanji S, Martinez V, Mand S, Adjei O, Bain O, Specht S, Martin C: Lymphatic vascularisation and involvement of Lyve- $1^{+}$macrophages in the human onchocerca nodule. PLoS One 2009, 4:e8234

28. Wang XN, McGovern N, Gunawan M, Richardson C, Windebank M, Siah TW, Lim HY, Fink K, Li JL, Ng LG, Ginhoux F, Angeli V, Collin M, Haniffa M: A three-dimensional atlas of human dermal leukocytes, lymphatics, and blood vessels. J Invest Dermatol 2014, 134:965-974

29. Böckle BC, Sölder E, Kind S, Romani N, Sepp NT: DC-sign ${ }^{+}$CD163 ${ }^{+}$ macrophages expressing hyaluronan receptor LYVE-1 are located within chorion villi of the placenta. Placenta 2008, 29:187-192

30. Gröger M, Niederleithner H, Kerjaschki D, Petzelbauer P: A previously unknown dermal blood vessel phenotype in skin inflammation. J Invest Dermatol 2007, 127:2893-2900
31. Wigle JT, Harvey N, Detmar M, Lagutina I, Grosveld G, Gunn MD, Jackson DG, Oliver G: An essential role for Prox 1 in the induction of the lymphatic endothelial cell phenotype. EMBO J 2002, 21:1505-1513

32. Zaba LC, Fuentes-Duculan J, Steinman RM, Krueger JG, Lowes MA: Normal human dermis contains distinct populations of CD11 $\mathrm{c}^{+} \mathrm{BDCA}-$ $1^{+}$dendritic cells and $\mathrm{CD} 163^{+} \mathrm{FXIIIA}^{+}$macrophages. J Clin Invest 2007, 117:2517-2525

33. Albrecht I, Christofori G: Molecular mechanisms of lymphangiogenesis in development and cancer. Int J Dev Biol 2011, 55:483-494

34. von Tell D, Armulik A, Betsholtz C: Pericytes and vascular stability. Exp Cell Res 2006, 312:623-629

35. Fujita M, Furukawa F, Horiguchi Y, Ueda M, Kashihara-Sawami M, Imamura S: Regional development of Langerhans cells and formation of Birbeck granules in human embryonic and fetal skin. J Invest Dermatol 1991, 97:65-72

36. Pober JS, Kluger MS, Schechner JS: Human endothelial cell presentation of antigen and the homing of memory/effector $\mathrm{T}$ cells to skin. Ann N Y Acad Sci 2001, 941:12-25

37. Nguyen A, Hoang V, Laquer V, Kelly KM: Angiogenesis in cutaneous disease: part I. J Am Acad Dermatol 2009, 61:921-942

38. Otberg N, Richter H, Schaefer H, Blume-Peytavi U, Sterry W, Lademann J: Variations of hair follicle size and distribution in different body sites. J Invest Dermatol 2004, 122:14-19

39. Coultas L, Chawengsaksophak K, Rossant J: Endothelial cells and VEGF in vascular development. Nature 2005, 438:937-945

40. Hellstrom M, Kalen M, Lindahl P, Abramsson A, Betsholtz C: Role of PDGF-B and PDGFR-beta in recruitment of vascular smooth muscle cells and pericytes during embryonic blood vessel formation in the mouse. Development 1999, 126:3047-3055

41. Goumans MJ, Valdimarsdottir G, Itoh S, Rosendahl A, Sideras P, Ten DP: Balancing the activation state of the endothelium via two distinct TGF-beta type I receptors. EMBO J 2002, 21:1743-1753

42. Hawighorst T, Skobe M, Streit M, Hong YK, Velasco P, Brown LF, Riccardi L, Lange-Asschenfeldt B, Detmar M: Activation of the tie2 receptor by angiopoietin- 1 enhances tumor vessel maturation and impairs squamous cell carcinoma growth. Am J Pathol 2002, 160:1381-1392

43. Isogai S, Lawson ND, Torrealday S, Horiguchi M, Weinstein BM: Angiogenic network formation in the developing vertebrate trunk. Development 2003, 130:5281-5290

44. Mancuso MR, Davis R, Norberg SM, O’Brien S, Sennino B, Nakahara T, Yao VJ, Inai T, Brooks P, Freimark B, Shalinsky DR, HuLowe DD, McDonald DM: Rapid vascular regrowth in tumors after reversal of VEGF inhibition. J Clin Invest 2006, 116:2610-2621

45. Inai T, Mancuso M, Hashizume H, Baffert F, Haskell A, Baluk P, Hu-Lowe DD, Shalinsky DR, Thurston G, Yancopoulos GD, McDonald DM: Inhibition of vascular endothelial growth factor (VEGF) signaling in cancer causes loss of endothelial fenestrations, regression of tumor vessels, and appearance of basement membrane ghosts. Am J Pathol 2004, 165:35-52

46. Kerjaschki D: The crucial role of macrophages in lymphangiogenesis. J Clin Invest 2005, 115:2316-2319

47. Wigle JT, Oliver G: Prox 1 function is required for the development of the murine lymphatic system. Cell 1999, 98:769-778

48. Karkkainen MJ, Haiko P, Sainio K, Partanen J, Taipale J, Petrova TV, Jeltsch M, Jackson DG, Talikka M, Rauvala H, Betsholtz C, Alitalo K: Vascular endothelial growth factor $\mathrm{C}$ is required for sprouting of the first lymphatic vessels from embryonic veins. Nat Immunol 2004, 5:74-80

49. Kim YM, Romero R, Chaiworapongsa T, Espinoza J, Mor G, Kim CJ: Dermatitis as a component of the fetal inflammatory response syndrome is associated with activation of Toll-like receptors in epidermal keratinocytes. Histopathology 2006, 49:506-514

50. Nucera S, Biziato D, De PM: The interplay between macrophages and angiogenesis in development, tissue injury and regeneration. Int J Dev Biol 2011, 55:495-503

51. Carmeliet P: Angiogenesis in health and disease. Nat Med 2003, 9: 653-660 